\title{
La manipulation des images par les femmes : à propos des Vierges miraculeuses de Marlène Albert Llorca
}

Richard C. Trexler

\author{
(2) OpenEdition \\ Journals \\ Édition électronique \\ URL : http://journals.openedition.org/clio/651 \\ DOI : 10.4000/clio.651 \\ ISSN : 1777-5299 \\ Éditeur \\ Belin
}

Édition imprimée

Date de publication : 1 avril 2004

ISSN : 1252-7017

Référence électronique

Richard C. Trexler, «La manipulation des images par les femmes : à propos des Vierges miraculeuses de Marlène Albert Llorca », Clio. Femmes, Genre, Histoire [En ligne], 19| 2004, mis en ligne le 08 novembre 2006, consulté le 03 mai 2019. URL : http://journals.openedition.org/clio/651 ; DOI : 10.4000/clio.651

Ce document a été généré automatiquement le 3 mai 2019.

Tous droits réservés 


\section{La manipulation des images par les femmes : à propos des Vierges miraculeuses de Marlène Albert Llorca}

Richard C. Trexler

1 Il n'y a pas si longtemps, la plupart des catholiques croyaient dans les pouvoirs miraculeux des images religieuses ; toutefois, peu d'études scientifiques leur avaient été consacrées. On ne disposait que d'opuscules de dévotion sur des images particulières, ou de vastes catalogues d'images mariales provenant de toute l'Europe, qui tous décrivaient les miracles accomplis par ces images de Marie. Remarquablement semblables, ces miracles montraient en général la Vierge s'adressant à des individus, pleurant des larmes ou du sang. Au siècle dernier, cependant, les manifestations de ces Maries furent moins le fait d'images ou de statues que d'apparitions à des dévots particuliers survenant à l'air libre. Grâce aux médias modernes, certains lieux gratifiés par ces apparitions sont rapidement devenus des objectifs du tourisme international dotés de tous les moyens à la page, sites web, etc. et les gens accourent " voir l'endroit où... ». Fatima, entre autres, Lourdes ou Medjugorje sont encore aujourd'hui immensément populaires.

2 Cette lente évolution des images aux apparitions miraculeuses a cependant éclipsé nombre de bourgades ou de villes en Europe dont les images légendaires en étaient venues, avant le tourisme de masse, à si bien s'identifier à la localité que la statue religieuse était prise pour totem civique. Dans ces localités, dont l'Espagne compte un bon nombre, l'histoire de la ville, voire du pays, se confond encore à peu près avec celle de leur image civique, et celle-ci à son tour confère à ces lieux un pouvoir d'attraction auquel les ethnographes modernes et les chercheurs intéressés par la soi-disant « religion populaire " ne peuvent résister. Car, tout comme les anthropologues sociaux venus d'Angleterre le découvraient naguère dans les tribus africaines, les chercheurs trouvent ici les traces de sociétés du passé où les actes religieux entourant des images constituaient l'essence même de l'organisation sociale et de l'identité civique. 
3 L'église catholique a tout à la fois dirigé et suivi ces développements. Depuis la seconde moitié du XVIe siècle, la Contre-Réforme a défié les attaques des protestants contre «l'adoration des images » en encourageant la propension des laïcs au culte de celles-ci, tout en insistant sur le fait que les catholiques les vénérent plutôt qu'ils ne les adorent. Ainsi, le temple protestant typique s'est rapidement distingué d'une église catholique en se dépouillant de presque toute image. Il a fallu attendre le concile de Vatican II (1962-1965) pour constater la victoire du protestantisme en la matière. Un garçon qui comme moi a été élevé dans le catholicisme avant le concile, a vu les vieilles statues disparaître l'une après l'autre des églises tandis que les nouveaux lieux de culte évitaient d'en incorporer ou en limitaient le nombre.

Ce processus a certainement été retardé dans l'Espagne de Franco. Mais à sa mort, en 1975, l'avenir était clairement tracé quand les jeunes prêtres commencèrent à appliquer les règles de Vatican II sur les images, suscitant un profond malaise chez beaucoup de leurs paroissiens, surtout dans les zones rurales d'un pays encore largement traditionnel. La constante opposition des gens du cru à cette nouvelle orientation entraîna cependant des compromis sensibles, de sorte que le visiteur pénétrant aujourd'hui dans plus d'une église de la péninsule ibérique peut encore voir le décor d'antan et participer à la vie festive traditionnelle qui entoure souvent ces statues ${ }^{1}$. L'ironie, aujourd'hui, est frappante pour l'historien. Car ces changements dans les églises sont intervenus en Espagne au moment même où dans la plus grande partie de l'Europe et de l'Amérique les images diffusées sur les écrans des médias modernes submergeaient la vieille méfiance protestante envers les images. Un univers d'images en affronte ainsi un autre.

5 En tout cas, la vie festive religieuse, dans la majeure partie de l'Espagne comme dans le sud de l'Italie, continue à se dérouler imperturbablement dans un monde toujours plus laïcisé, encore que soumis à la nouvelle vogue de l'image télévisée. Ce qui, à son tour, a suscité l'intérêt d'éminents chercheurs capables d'exploiter et d'expliquer les fêtes traditionnelles sans les engloutir dans l'histoire des dogmes et des sentiments -l'histoire de la soi-disant spiritualité. Leurs travaux enrichissent notre compréhension des mentalités traditionnelles en Europe qui nous restent si difficilement accessibles. Dans la continuité du travail de Manuel Trens, la photographe Cristina Garcia Rodero a ouvert la voie en rassemblant une collection impressionnante de photographies de fêtes et en en publiant deux recueils ${ }^{2}$. Parmi les historiens, il faut citer Gabriel Llompart, Maureen Flynn et Antonio Cea Guttiérrez ${ }^{3}$. De son côté William Christian a interrogé la documentation à la fois en anthropologue et en historien et il est arrivé à des conclusions stimulantes ${ }^{4}$. D'autres groupes de chercheurs ont publié les actes de colloques importants, dont trois par exemple portent sur les activités de la Semaine sainte ${ }^{5}$. Écrivant pour un public moderne, mais flattant le public traditionnel en signalant leurs localités aux habitants des grandes villes, ces chercheurs disposent d'énormes ressources: archives locales et bibliothèques croulant sous les imprimés anciens qui justifient miracles et sanctuaires; à tous les coins du bourg, ils tombent sur des vendeurs de cartes postales holographiques d'occasion qui permettent de vérifier le normal et le paranormal dans les statues et les apparitions miraculeuses; et surtout, ils rencontrent des habitants ne désirant qu'une chose : partager avec des étrangers complaisants leurs souvenirs des miracles passés. L'Espagne n'a peut-être pas encore trouvé son Van Gennep pour mettre un peu d'ordre dans le trésor de ses traditions, mais elle a certainement bénéficié de bien d'autres chercheurs capables de décrire des pans importants de cet univers dévotionnel, certes en voie de disparition, mais encore bien vivant. 
6 Le livre de Marlène Albert-Llorca, Les Vierges miraculeuses, est un apport important à cet ensemble d'études sur les dévotions espagnoles. Les yeux grand ouverts, l'auteur étudie l'usage de la statuaire traditionnelle (il y a peu d'images peintes au palmarès des miracles) dans deux régions distinctes de l'Espagne. La première couvre des localités sises pour une grande part au nord-ouest de Gérone, en Catalogne ${ }^{6}$; la seconde englobe l'aire entre Valence et Alicante ${ }^{7}$. L'auteur recourt abondamment aux légendes écrites, dont certaines remontent aux débuts de l'époque moderne et qui relatent la « découverte » de chaque statue. À en croire les fidèles, on aurait d'habitude trouvé ces statues anonymes sous un arbre ou dans l'eau et on en a fréquemment attribué l'origine à saint Luc. En fait, leur localisation était souvent un moyen d'élargir ou de redessiner la géographie politique locale. L'auteur combine alors ces mémoires écrits de l'événement avec ceux plus modernes du rituel qui s'organise autour de la statue, ainsi qu'avec les interviews par lesquelles elle a tenté de saisir le sens qu'avaient ces rituels pour les participants. C'est ici que ses trouvailles sont les plus originales.

7 L'ouvrage se divise en cinq parties d'importance inégale. La première partie, intitulée « La naissance des images ", s'avère d'un grand intérêt pour le sujet. L'auteur y discute la prétention fréquente à voir dans une statue donnée un "vrai portrait» de la Vierge, expliquant comment on peut ainsi la considérer ici ou là comme une relique. Elle analyse ensuite le topos du berger qui vient en ville raconter sa découverte d'une statue; et elle finit par des considérations sur la manière dont les paroissiens distinguent leur Vierge de celle d'une paroisse voisine.

Dans la seconde brève partie intitulée "Le jardin de Marie ", j'ai eu du mal à saisir le rapport avec le thème général du livre, bien que l'arbre de vie aux racines duquel Marie est assise en relève partiellement. Mais il n'a pas grand chose à voir avec le rituel ou l'utilisation de la statuaire et des apparitions. La troisième partie qui, en revanche, est solide et bien centrée, discute le rôle de ces statues dans la « religion civique ». L'auteur y aborde les implications purement intellectuelles de conflits inhérents à cette religion civique, le fait par exemple que chaque communauté politique ou paroisse a sa Vierge propre («à chacun sa Vierge»), appelée par les paroissiens « Notre Dame», si bien que chacune des Vierges Marie entre en compétition avec une ou plusieurs Vierges du même nom et de semblable identité. Mais ce qui fait l'objet du culte, comme l'admet Marlène Albert-Llorca, est non pas "la Vierge", mais l'image particulière de la ville et son histoire. L'auteur aborde alors le thème central des vêtements de la Vierge, de sa couronne, de ses déplacements, et termine sur le cas particulier de la "Vierge des Desamparats » valencienne, dont le « corps » et les habits consistent presqu'entièrement en fleurs.

La quatrième partie, tout aussi importante, est appelée "Dans l'intimité de la Vierge ». L'auteur y décrit chacune des pièces du vêtement de la Vierge, en la déshabillant progressivement comme le ferait un sacristain. Elle interviewe alors quelques-unes des femmes, les cameraras, responsables de l'habillement et du déshabillage de la statue, testant leurs réactions lorsqu'elle s'avise que leur Vierge n'a pas de corps sous ses habits. C'est pour moi le point fort du livre, et je vais y revenir. Et d'autant plus volontiers, ajouterai-je, que je n'ai pas saisi comment la dernière partie, «Comme une rose au milieu des épines ", se rattache au cœur de l'ouvrage, qui traite des conduites rituelles. Cette partie présente des motifs iconographiques tels que le Buisson ardent et le Rosaire. Bon nombre des images qui illustrent excellemment le livre concernent cette dernière partie. 
10 On doit donc dire que ce livre de grande valeur souffre d'un certain manque d'intégration, ce que met aussi en lumière le fait qu'en dépit de son titre il ne décrit réellement aucun des faits "miraculeux » qu'une Vierge quelconque est censée avoir accomplis, si l'on excepte sa "découverte ». L'auteur, en effet, n'explicite pas ce qu'elle, ou ses protagonistes, considèrent comme une Vierge " miraculeuse » et, de fait, d'aucune de ces Vierges il n'est dit qu'elle ait sauvé telle ville de la peste ou guéri telle dame de son hydropisie ou accompli tel haut fait historique. Il est vrai qu'à un moment l'auteur semble admettre qu'elle n'étudie pas ces images parce qu'elles sont dites miraculeuses, mais parce qu'elle sont vénérées comme des talismans civiques (p. 148).

Plusieurs passages du livre méritent une attention particulière. Le premier consiste en la découverte d'une nouvelle tradition jusqu'ici inconnue, ou que pour ma part du moins je ne connaissais pas. "Récemment", écrit Marlène Albert-Llorca, des communes ont décidé de donner une représentation annuelle, de type théâtral (pour nous sinon pour elles), de la découverte locale d'une statue, à l'anniversaire non pas de la découverte mais de l'annonce de celle-ci à la ville, annonce également représentée sous forme théâtrale. Selon l'auteur, des parties de l'histoire sont jouées par des descendants des découvreurs, si bien que, plutôt que d'une production purement professionnelle, on peut parler ici d'une sorte de « liturgie laïque » rappelant les défunts des familles et de la cité en faisant revivre leur passé (p. 152). À la fin du Moyen Âge il était courant que des villes commémorent une victoire, par exemple, en la rejouant non pas à son jour anniversaire, mais, de façon très égocentrique, à celui où la nouvelle leur en était parvenue ${ }^{8}$.

Une autre conclusion de notre auteur évoque la possibilité qu'en voilant et dévoilant des images, les dévots aient été amenés à revivre une apparition vécue jadis par un membre de la communauté - en un autre type de remémoration d'une expérience. Encore une fois, cette constatation importe particulièrement à l'historien, car le dévoilement des images a eu une importance toute spéciale dans les dévotions du Moyen Âge tardif, en Italie, même si les sources n'indiquent pas que les gens aient dit voir réellement la Vierge, et non pas simplement son image, lorsqu'elle était temporairement dévoilée ${ }^{9}$. Marlène Albert-Llorca nous convainc en tout cas que quelques fidèles, même dans le monde contemporain, voient dans cette technique rituelle une sorte d'accès " démocratisé " à l'expérience visionnaire - un peu comme telle dévote à Lourdes affirmant avoir vu, à l'instar de Bernadette, la Vierge sortir de dessous son voile! Ici, à l'approche du moment du dévoilement, les croyants s'écrient : « Nous allons voir la Vierge, nous allons voir la Vierge!» Et, lorsqu'elle est dévoilée: "Je l'ai vue!»(p. 54-62). Selon notre auteur, chacun peut re-voir cette Vierge en vivant la même expérience que fit son premier découvreur. Suivant ce fil directeur, il me semble que les historiens devraient remonter le temps pour vérifier si cette technique historisante démocratisée est à l'œuvre chez les croyants du Moyen Âge.

13 Un troisième pan du travail de l'auteur qui mérite une attention spéciale concerne ses interviews des cameraras qui habillent et déshabillent les statues, et c'est de loin la partie la plus originale du livre. En s'appuyant en particulier sur le récent travail d'une équipe italienne menée par Riccarda Pagnozzato ${ }^{10}$, Marlène Albert-Llorca a réussi à intégrer le phénomène italien de l'habillage-déshabillage des images, qui remonte au moins au XIVe siècle, au modèle ibérique mieux connu. Ici, des cameraros mâles occasionnels, que l'auteur considère comme des techniciens, ont fait place à l'époque moderne à un corps entièrement féminisé, qu'elle caractérise comme constitué de dévotes. Dans ses interviews de quelques cameraras, elle pose des questions intimes, ce que, chercheur 
femme, elle pouvait plus aisément faire, et elle le fait fort bien. Si limités que soient les questions et le nombre des informatrices, ces interviews me paraissent un type de source à exploiter impérativement avant que ces femmes et leur institution aient disparu. Je m'explique. Une camerara dit que cela fera peut-être rire l'enquêtrice, mais que ses camarades pensent comme elle qu'il est important de parfumer Marie « afin qu'elle sente bon» (p. 150). L'influence de la culture établie est ici sensible. L'auteur explique que, si jadis seules les femmes âgées les plus respectables pouvaient devenir cameraras, toutes les femmes aujourd'hui ont droit à la fonction; après un détour, toutefois, elle ajoute que cela n'arrive que si elles peuvent payer les dépenses plutôt élevées que cette fonction entraîne (p. 136-137). Une autre femme explique pourquoi elle aime habiller la Vierge : il lui semble alors qu'" elle était à nous ", dit-elle (p. 160). Une troisième décrit ce qu'elle ressent quand elle participe au déshabillage et au rhabillage de Notre Dame: "C'est une statue mais, pour moi, ce n'est pas une statue. Alors, l'habiller, c'est une joie, c'est... C'est un sentiment qu'on ne peut pas expliquer. C'est bouleversant parce que c'est une chose si grande. C'est... Toute l'année, chaque fois qu'on a un problème, on est là à dire : “Aïe, Très Sainte Vierge". Et puis, venir, et être là à la toucher...» (p. 161) Voilà un discours signifiant et combien précieux !

14 Mais comment les cameraras affrontent-elles le fait qu'il ne s'agisse que d'une statue, "voire d'un mannequin", demande notre enquêtrice. "La Vierge n'est pas qu'un mannequin et les cameraras ont peur que nous [les femmes qui mettent les bijoux] nous moquions d'elle ", répond une informatrice (p. 161). Une autre affirme qu'elle n'aime pas avoir quelqu'un à côté d'elle quand elle habille la Vierge, parce que celle-ci «fait trop poupée » (p. 163). Mais « quand j'ai fini de l'habiller, elle me fait presque peur. Elle a l'air vivante, vous comprenez » (p. 163), Marlène Albert-Llorca cite à ce propos les dires bien connus de la grande Pasionaria, Dolores Ibarruri, racontant comment ceux qui par inadvertance s'apercevaient que leur Vierge était une effigie "sans corps et sans cheveux » pouvaient en être assez bouleversés pour se détourner, comme elle-même, du catholicisme (p. 162).

15 Aussi les cameraras prennent-elles leurs dispositions. « Nous nous arrangions pour ne pas voir comment la Vierge était faite : à mesure que nous faisions descendre la chemise, nous enfilions l'autre ». Q. : «Et donc vous ne saviez pas que la Vierge était vide? » R. : " $\mathrm{Si}$, parce que nous le sentions en la touchant. Mais nous faisions tout pour ne pas voir " (p. 161-164). Paroles révélatrices! Mais des échanges aussi brefs nous laissent sur notre faim : l'auteur n'aurait-elle pu pousser plus avant? Car ces femmes devaient ruminer encore plus profondément, plus anxieusement le problème. Mais comme ces échanges sont excitants! Il faudra les poursuivre par des enquêtes s'engageant plus loin dans l'interprétation.

16 Si précieuses que soient ces pages, l'auteur ne passe malheureusement pas du sexe au genre, si je peux dire, et se contente en général de l'idée qu'il semblait raisonnable aux prêtres (définis à tort comme des super-mâles) ou aux femmes d'accomplir un travail qui aujourd'hui est limité à celles-ci. Ainsi, elle ne mentionne ni n'affronte le fait important qu'au moins dans une localité d'Andalousie proche de Séville (Castilleja de la Cuesta), des gays ou du moins des travestis laïques (les maricas) sont les seuls qui habillent les Señoras, probablement parce que beaucoup les considèrent, selon le stéréotype féminin, habiles dans l'art de la parure ${ }^{11}$. Reste, malgré ces quelques réserves, que l'ouvrage de Marlène Albert-Llorca est une contribution importante et novatrice à l'historiographie de la vie dévotionnelle en Espagne. 


\section{BIBLIOGRAPHIE}

BEHAR R. , 1990, « The Struggle for the Church: Popular anticlericalism and religiosity in postFranco Spain ", in Religious Orthodoxy and Popular Faith in European Society, ed. E. Badone, Princeton, Princeton University Press, pp. 76-112.

BRETTELL C. , 1990, «The priest and his people: The contractual basis for religious practice in rural Portugal ", in Religious Orthodoxy and Popular Faith in European Society, ed. E. Badone, Princeton, Princeton University Press, pp. 55-75.

CEA GUtIÉRREZ Antonio, 1983, Religiosidad popular : Imàgines vestideras, cat. expo., Zamora, Caja España-Obra Cultural.

CHRISTIAN William A., 1981, Local religion in sixteenth-century Spain, Princeton, Princeton University Press.

CRIADI Encarnación, 1983, Las Hermandades de Castilleja de la Cuesta, Séville, Servicio de publicaciones del Ayuntamiento.

España oculta: Public celebrations in Spain, 1974-1989, 1995, Washington, D. C., Smithsonian Institute. FLYNN Maureen, 1989, Sacred charity: Confraternities and social welfare in Spain, 1400-1700, Ithaca, Cornell University Press.

LlOMPART Gabriel, 1984, Entre la Historia del Arte y el Folklore, Palma de Mallorca, Fontes Rerum Balearium.

PAGnOzZATo Riccarda (dir.), 1993, Madonne della laguna : Simulacri ‘da vestire’ dei secoli XIV-XIX, Rome.

TRENS M., 1946, Maria. Iconografia de la Virgen en el arte espanol, Madrid.

- 1966, Les Majestats catalanes, Barcelone.

TREXLER Richard C., 1980, Public life in Renaissance Florence, New York, Academic Press.

- 1991, « Habiller et déshabiller les images. Esquisse d'une analyse », in F. Dunand, J.-M. Spieser et J. Wirth (dir.), L'Image et la production du sacré, Paris, Klincksieck, pp. 195-231.

- 1995, Sex and Conquest. Gendered violence, political order, and the European conquest of the Americas, Ithaca et New York, Cornell University Press.

\section{NOTES}

1. Particulièrement utiles : Brettell 1990 et Behar 1990.

2. Trens 1946 ; Les Majestats catalanes 1966 ; pour Garcia Rodero, voir Espana oculta 1995.

3. Llompart 1984 ; Flynn 1989 ; Cea Guttiérrez 1992.

4. Christian 1981.

5. I. Congreso nacional de confradias de Semana Santa, Zamora : Diputacial de confradias de Semana Santa.

6. Font-Romeu et St-Laurent-de-Cerdans en France, Nùria, Queralbs, Coustouges, Na Sa del Mont, Maia, Besalù en Espagne. 
7. Albuixech, Algemesi, Alzira, Carcaixent, Agres, Muro del Alcoy, Caudete, Villena, Biar, Aspe, Elche et Mondo de la Nieves.

8. Sur ces attitudes envers les saints et les temps, voir Trexler $1980: 55-56,77$ et suiv.

9. Trexler 1991.

10. Pagnozzato 1993.

11. Criadi $1983: 122-127,179-180$; cité par Trexler $1995: 134-137$.

\section{RÉSUMÉS}

À propos du livre récent de Marlène Albert Llorca, Les Vierges miraculeuses, l'auteur survole rapidement les conclusions de recherches récentes sur l'image religieuse dans l'Europe traditionnelle. Il examine les manières dont les dévots convertissent des images en objets cultuels en les associant à des laïcs importants et aux sanctuaires qui les accueillent. Il commente les conclusions d'Albert Llorca selon lesquelles ces images, vêtues pour la circonstance, peuvent servir à répliquer le "miracle" originel pour les dévots qui manipulent ces vêtements ; ainsi se rejouent les événements miraculeux censés être à l'origine du charisme de l'image. C'est donc l'imaginaire de la communauté, incarné dans les "cameraras" qui habillent l'image, qui dote celle-ci de pouvoirs miraculeux.

Inspired by the recent book of M. Albert-Llorca, the author presents a rapid overview of some of the findings behind recent studies of the traditional European religious image. He examines the process by which devotees convert images into cult objects through their association with powerful lay figures and with the important churches that receive them. He comments upon M. Albert-Llorca's findings that such church images, suitably attired, can serve to replicate for devotees the original "miracle" of the image by manipulation of the image's veil. And he elaborates as well on M. A. L.'s finding that at least modern devotees re-stage the "miraculous" events said to lie at the origins of the image's charisma. Clearly, no image is in fact miraculous, it is the imagination of the community, embodied in the "cameraras" who dress it, that endows it with wonder.

\section{INDEX}

Mots-clés : apparitions, dévotion, habillement, statues, vierge Marie

Index géographique : Espagne

\section{AUTEUR}

\section{RICHARD C. TREXLER}

Richard C. TREXLER, docteur de la Goethe Universität de Francfort/Main, a enseigné dans les universités du Texas, Los Angeles, Illinois et Binghamton dont il est aujourd'hui professeur d'histoire émérite. Il est l'auteur de plusieurs livres et articles sur l'histoire sociale et religieuse de l'Italie et plus généralement de l'Europe ainsi que du Nouveau Monde, entre autres : Public Life 
in Renaissance Florence (New York et Londres, Academic Press, 1980); The Christian at Prayer. An illustrated Prayer Manual Attributed to Peter the Chanter (d. 1197), (Binghamton, 1987); Sex and Conquest. Gendered Violence, Political Order and the European Conquest of the Americas (Ithaca, Cornell University Press, 1995); Religion in Social Context in Europe and America, 1200-1700 (Tempe, Arizona State University, 2002). Il travaille à un livre sur l'émigration forcée des enfants, 1492-1970. 